\title{
Frecuencia de Mycoplasma pneumoniae y Chlamydia pneumoniae en pacientes con distress respiratorio y serología negativa para hantavirus
}

MARITZA NAVARRETE C. ${ }^{1-2}$, MYRA WILSON S. ${ }^{2}$, LAURA OTTH R. ${ }^{2}$,

IGNACIO HOFFMAN H. ${ }^{2}$ y LUIS ZAROR C. ${ }^{2}$

\section{Mycoplasma pneumoniae and Chlamydia pneumoniae frequency in respiratory distress syndrome with negative serology for hantavirus}

A retrospective study by means of indirect immunofluorescence was carried out to detect $\operatorname{IgM}$ antibodies against Chlamydia pneumoniae and Mycoplasma pneumoniae in seronegative patients for Hantavirus, with characteristics symptoms of atypical pneumonia. The final results indicate that the prevalence of $C$. pneumoniae reaches $8,6 \%$ and M. pneumoniae is equivalent to $17,1 \%$ of the patients studied. By this we can be aware of the importance of these agents in our media, and the necessity of having rapid laboratory techniques that allow an opportune differential diagnosis in pulmonary syndrome for Hantavirus and other pathologies that cause similar symptoms, mainly atypical pneumonia.

Key words: Mycoplasma, Chlamydia, atypical pneumonia, distress.

\section{Introducción}

En E.U.A. se describió por primera vez en 1993, un síndrome de dificultad respiratoria aguda producido por hantavirus en pacientes previamente sanos, caracterizado por un pródromo "gripal" con calofríos, cefalea, síntomas gastrointestinales, seguido de compromiso respiratorio rápidamente progresivo, que requirió oxigenación suplementaria y que evolucionó como un distress respiratorio del adulto (SDRA), letal en $52 \%$ de los $\operatorname{casos}^{1}$. En Chile la infección por hantavirus representa un problema de salud pública desde 1995, fecha en la que se detectaron los primeros casos de síndrome pulmonar por hantavirus (SPH), estableciéndose en forma retrospectiva la presencia de esta enfermedad ya en $1993^{2}$. A la fecha (7 de marzo, 2003) se han diagnosticado 289 casos de la infección desde la $\mathrm{V}$ a la XI Región, con una letalidad global de $40 \%{ }^{3}$.

Los síntomas y signos del SPH son similares a otros cuadros clínicos como gripe, gastroenteritis, neumonías típicas, neumonías atípicas, sepsis, entre otros, por lo que se hace necesario establecer el diagnóstico diferencial en aquellos casos con serología negativa para hantavirus.

Los patógenos atípicos más comunes son Mycoplasma pneumoniae y Chlamydia pneumoniae considerados como una importante causa de neumonías adquiridas en la comunidad (NAC), siendo también responsables de un amplio espectro de manifestaciones extrapulmonares que incluyen compromiso neurológico, hepático, cardíaco y hematológico. Las tasas de infección para estos agentes no están bien establecidas por la falta de confirmación diagnóstica, ya que la mayoría de los tratamientos son empíricos ${ }^{4,5}$.

El objetivo del presente trabajo fue determinar la frecuencia de infección por M. pneumoniae y $C$. pneumoniae en cuadros de SDRA, con serología negativa para hantavirus, analizados en el Laboratorio de Virología del Instituto de Microbiología Clínica de la Universidad Austral de Chile.

\footnotetext{
Hospital Clínico Regional Valdivia.

Instituto de Microbiología Clínica, Facultad de Medicina, Universidad Austral de Chile.
}

Recibido: 22 abril 2002

Aceptado: 14 diciembre 2002 


\section{Material y Método}

Muestras clínicas: Se seleccionó 35 de 107 sueros negativos en forma aleatoria para hantavirus (IgM mediante ELISA de captura empleando como antígeno virus Laguna Negra) provenientes de pacientes con diagnóstico de sospecha de SPH, y que presentaban fiebre, mialgias, síntomas respiratorios y radiografía de tórax con infiltrados intersticiales. Los datos fueron extractados del formulario de notificación inmediata y envío de muestras para casos sospechosos de infección por hantavirus (MINSAL). La distribución etaria fue de 2 a 88 años.

Estudio serológico específico para M. pneumoniae y $C$. pneumoniae. Tanto para $C$. pneumoniae como $M$. pneumoniae, se investigó la presencia de anticuerpos IgM en el suero de los pacientes mediante IFI. Se eligió esta técnica porque no se contaba con muestras pareadas y como lo describe la literatura, la presencia de ella es indicativa de infección actual o reciente ${ }^{6-10}$.

Chlamydia pneumoniae: Se utilizó un kit de IFI (Fuller Laboratories). Las placas de IFI utilizan como sustrato antigénico, células de cultivo Hep 2 en los cuales se ha propagado C. pneumoniae y se encuentran por lo tanto con cuerpos elementales. El suero del paciente fue inicialmente diluido para screening en una solución diluyente de $\operatorname{IgM}(1: 16)$ en la cual los anticuerpos IgG fueron removidos por inmunoprecipitación selectiva. Luego de una incubación apropiada, las láminas se lavaron con el fin de remover las proteínas séricas no reactivas y enseguida se agregó un conjugado fluorescente anti IgM humana. Este conjugado se unió al complejo antígeno-anticuerpo y la reacción resultante se visualizó con un microscopio de fluorescencia, observándose en caso positivo, los cuerpos elementales intensamente teñidos verde manzana en el citoplasma en 10 a 20\% de las células infectadas.

Mycoplasma pneumoniae. Se utilizó el kit IgM Antibody Test System de Zeus Scientific, Inc. La técnica de IFI utiliza en este caso un sustrato antigénico de $M$. pneumoniae fijado en los pocillos de un portaobjeto. El suero a testar se diluyó 1:8 y se incubó con el sustrato y si el anticuerpo estaba presente, se pudo observar fluorescencia después de haber teñido con un conjugado fluorescente anti IgM humana. A las muestras positivas se les hizo, posteriormente, diluciones 1:16 y 1:32, para confirmar la positividad.

Tanto para $C$. pneumoniae como para $M$. pneumoniae se consideró la muestra positiva si se observó fluorescencia a un título de 1:16 o más.

\section{Resultados}

En nuestro estudio, de 35 sueros negativos para hantavirus, $3(8,6 \%)$ y $6(17,1 \%)$ de estos tenían anticuerpos del tipo IgM anti $C$. pneumoniae y anti $M$. pneumoniae respectivamente, con títulos $\geq 1 / 16$ (Tabla 1 ). No se detectó una distribución etaria de los seropositivos.

\section{Discusión}

En nuestro estudio en $25,7 \%$ de los sueros estudiados se encontró anticuerpos del tipo IgM para M. pneumoniae o C. pneumoniae. El 8,6\% de los sueros (Tabla 1) presentó anticuerpos IgM anti $C$. pneumoniae con título $\geq 1 / 16$. Este resultado es similar al reportado por otros autores, quienes informan a $C$. pneumoniae como responsable de 6 a 10 y hasta $15 \%$ de las neumonías adquiridas en la comunidad ${ }^{711-13}$. No encontramos datos de la incidencia de infecciones por $C$. pneumoniae en Chile. Sin embargo, Martínez et $\mathrm{al}^{14}$ reportan $60 \%$ de seroprevalencia en individuos asintomáticos, sugiriendo que la infección por C. pneumoniae en Chile es endémica. Peeling y Brunham ${ }^{12}$, sostienen que ello representa un problema de salud publica al establecer que entre 50 y $70 \%$ de las infecciones son clínicamente silentes.

Gran parte de la información epidemiológica de la infección por $C$. pneumoniae deriva de estudios serológicos específicos por inmunofluorescencia. Estos estudios indican que $C$. pneumoniae es un agente frecuente de infección en el mundo, con una seroprevalencia mayor al $50 \%$ en adultos en E.U.A. y otros países. Neumonía y bronquitis son los cuadros clínicos mayormente asociados a C. pneumoniae, alcanzando a 6 a $10 \%$ de las $\mathrm{NAC}^{12}$. La infección es frecuentemente leve o asintomática pero puede ser severa, especialmente en el anciano, probablemente favorecida por enfermedades de base, daño del clearence mucociliar y senescencia inmunológica. Casos de infección extrapulmonar

Tabla 1. Número y porcentaje de muestras positivas para anticuerpos IgM anti Mycoplasma pneumoniae y Chlamydia pneumoniae. Valdivia, Chile.

\begin{tabular}{lcc}
\hline Microorganismos & $\mathbf{N} / \mathbf{n}$ & $\%$ \\
\hline Mycoplasma pneumoniae & $6 / 35$ & 17,1 \\
Chlamydia pneumoniae & $3 / 35$ & 8,6 \\
\hline
\end{tabular}


severa por $C$. pneumoniae han sido esporádicamente reportados como, eritema nodoso, síndrome de Guillain Barre, endocarditis con cultivo negativo, tiroiditis, artritis, encefalitis y meningoencefalitis ${ }^{8,15-17}$.

La infección es más común en niños de 5 a 14 años de edad y la mayoría de los adultos tiene evidencia serológica de infección antigua. La prevalencia aumenta dramáticamente desde los 5 años; a los 20 años aproximadamente el 50\% de las personas tiene niveles detectables de anticuerpos contra $C$. pneumoniae. La seroprevalencia continúa aumentando y alcanza el $75 \%$ en la población anciana. Estas tasas de prevalencia existen a pesar que la primera infección induce una respuesta de anticuerpos limitada (3 a 5 años), sugiriendo que la mayoría de las personas se reinfecta a lo largo de la vida ${ }^{7}$.

Se han identificado dos patrones de respuesta de anticuerpos a infección aguda por $C$. pneumoniae, uno está asociado a infección primaria y el otro a reinfección. En la infección primaria se detectan anticuerpos específicos por FC, apareciendo a las tres semanas de iniciada la enfermedad, IgM medible por micro IF Twar. IgG puede no aparecer hasta 6 a 8 semanas del inicio. En la reinfección la IgM puede no aparecer o estar con títulos bajos, aumentando rápidamente en 1 a 2 semanas, comienza a caer dentro de 2 meses y generalmente desaparece 4 a 6 meses después de la infección aguda. La IgG persiste y puede ser detectada aún a 3 o más años de ocurrida la infección aguda. Es recomendable remover la IgG del factor reumatoide ${ }^{7}$, ya que según Peeling et $a{ }^{18}$ pueden fallar en definir falsos positivos por IgM.

El análisis de una segunda muestra en el período de convalecencia es necesario para establecer diagnóstico. Siendo éste un estudio retrospectivo, no fue posible obtener en estos pacientes una segunda muestra, por lo que se optó por detectar IgM.

En relación a $M$. pneumoniae $17,1 \%$ de los sueros fueron positivos para $\operatorname{IgM}$ (título $\geq 1 / 16$ ). Resultados semejantes han sido reportados por otros autores ${ }^{4,19}$. Mycoplasma pneumoniae es agente etiológico de infecciones respiratorias altas y bajas de variada intensidad, más común en pacientes entre 5 y 9 años de edad ${ }^{13}$ de éstas sólo 3 a $5 \%$ corresponden a neumonías ${ }^{20}$, siendo también responsable de un amplio espectro de manifestaciones extrapulmonares, que incluyen enfermedad neurológica (neuropatía óptica, encefalitis, meningitis) ${ }^{21-25}$, dermatológica (síndrome de Stevens Johnson), hematológica (trombocitopenia), esplenomegalia, coagulación intravas- cular diseminada, anemia hemolítica ${ }^{26}$, gastrointestinal (vómitos, diarrea, pancreatitis), renal (nefritis intersticial, glomerulonefritis), hepática, cardíaca (pericarditis, miocarditis) ${ }^{27}$. La sospecha precoz de esta enfermedad puede facilitar el diagnóstico temprano y por lo tanto el inicio precoz y apropiado de tratamiento que puede modificar el curso de la enfermedad ${ }^{28-31}$.

En una serie clínica de 11 casos fatales de infección por $M$. pneumoniae se describen 10 casos con neumonía, 5 de 11 desarrollaron trombosis vascular, asociada a infarto en sitios como pulmón, bazo, riñón y cerebro. La coagulación intravascular diseminada ha sido asociada con infección por $M$. pneumoniae tanto en casos fatales como no fatales. Puede haber anemia hemolítica Coombs positivo, sugiriendo un desorden autoinmune. En la serie referida, el diagnóstico de infección por $M$. pneumoniae fue establecido por crioaglutininas, FC e IFI positiva, y en cuatro casos, por cultivo ${ }^{28}$.

Llamamos la atención respecto del conocimiento deficiente de la epidemiología y las manifestaciones clínicas de estas dos infecciones y su diagnóstico no se tiene en cuenta, debido entre otras causas a lo difícil de cultivar que son Chlamydia y Mycoplasma. Sin embargo, es importante, establecer el diagnóstico, ya que según Fang et $\mathrm{al}^{29}, 13,9 \%$ de los pacientes con neumonía muere en el hospital, aun cuando sólo fallecen 4,5 y $0 \%$ de los casos debidos a $C$. pneumoniae y $M$. pneumoniae respectivamente.

\section{Conclusión}

Se hace necesario ampliar el abanico de exámenes para otros agentes en el estudio etiológico de pacientes con síntomas respiratorios bajos e infiltrados pulmonares, procedentes de áreas endémicas para infección por hantavirus, contribuyendo a un mejor diagnóstico diferencial y evitando así tratamientos empíricos inadecuados.

\section{Resumen}

Se realizó un estudio retrospectivo por medio de IFI, para detectar anticuerpos IgM anti Chlamydia pneumoniae y Mycoplasma pneumoniae, en pacientes seronegativos para hantavirus, con sintomatología de neumonía atípica y distress respiratorio. Chlamydia pneumoniae alcanzó una prevalencia de $8,6 \%$ y $M$. pneumoniae de $17,1 \%$ en los pacientes estudiados. Se enfatiza la importancia que estos agentes tienen en nuestro medio 
y la necesidad de contar con técnicas de laboratorio rápidas, que permitan un diagnóstico diferencial oportuno entre el síndrome pulmonar por hantavirus y otras patologías que producen cuadros similares, principalmente con neumonía atípica.

\section{Bibliografía}

1.- Khan A S, Khabbaz R F, Armstrong L R et al. Hantavirus pulmonary syndrome: The first 100 cases. J Infect Dis 1996; 173: 1297-303.

2.- Navarrete M, Saldías F, Mancilla R, Zaror L, Ferres M. Evidencia clínicoepidemiológica de la existencia de síndrome pulmonar por hantavirus en Valdivia-Chile desde 1993. Rev Chil Infect 2000; 17: 233-40.

3.- MINSAL: Http://epi.minsal.cl/Reportes/Hantavirus/ Hantavirus.htm

4.- TAN J S. Role of atypical pneumonia pathogens in respiratory tract infections. Can Respir J 1999; 6 (A): 15-9.

5.- Smith R, Viatar L. Neurologic manifestations of Mycoplasma pneumoniae infections: diverse spectrum of diseases. A report of six cases and review of the literature. Clin Pediatr (Phila) 2000; 39: 195-201.

6.- Carter J B, Carter S L. Acute-phase, indirect fluorescent antibody procedure for diagnosis of Mycoplasma pneumoniae infection. Ann Clin Lab Sci 1983; 13: $150-55$.

7.- Kuo C C, Jackson L A, Campbell L A, Grayston T. Chlamydiae pneumoniae (TWAR). Clin Microbiol Rev 1995; 8: 451-61.

8.- Blasi F. Clinical features of Chlamydia pneumoniae acute respiratory infection. Clin Microbiol Infect 1996; 1 (Suppl 1): 14-118.

9.- Roitt I M, Brostoff J, Male D K. Inmunología 1997; $4^{\circ}$ ed.: 4.1 - 4.2, Harcourt Brace, Madrid.

10.- Jackson L A, Grayston T. Chlamydia pneumoniae En Mandell, Douglas and Bennett's: Principles and Practice of Infectious Diseases. Mandell G L, Bennett J E, Dolin R, eds $5^{\circ}$ ed 2000, Churchill Livingstone, Philadelphia, pp: 2007-14.

11.- Grayston J T. Infections caused by Chlamydia pneumoniae strain TWAR. Clin Infect Dis 1992; 15: 757-63.

12.- Peeling R W, Brunham R C. Chlamydiae as pathogens: New species and new issues. Emerg Infect Dis 1996; 2: 307-17.

13.- Bartlett J G, Breinam R F, Mandell L A, FILE T M. Community-acquired pneumonia in adults: Guidelines for management. Clin Infect Dis 1998; 26: 811-38.

14.- Martínez M A, Kogan R, Silva J J et al. Seroprevalence of Chlamydia pneumoniae in Chile. Scand J Infec Dis 1999; 31: 103-4.

15.- Socan M, Beovic B, Kese D. Chlamydia pneumoniae and meningoencephalitis. N Engl J Med 1994; 331: 406.

16.- Gieffers J, Reusche E, Solbach W et al. Failure to detect Chlamydia pneumoniae in brain sections of Alzheimer disease patients. J Clin Microbiol 2000; 38: 881-2.

17.- Saikku P. Epidemiologic association of Chlamydia pneumoniae and atherosclerosis: The initial serologic observation and more. J Infect Dis 2000; 181 (Suppl.3): S411-13.

18.- Peeling R W, Wang S P, Grayston J T et al. Chlamydia pneumoniae serology: Interlaboratory variation in microimmunofluorescence assay results. J Infect Dis 2000; 181 (Suppl 3): S426-9.

19.- Dorigo-Zetsma J W, Zaat SAJ, Van Wertheim PME et al. Comparison of PCR, culture, and serological test for diagnosis of Mycoplasma pneumoniae respiratory tract infection in children. J Clin Microbiol 1999; 37: 14-7.

20.- Noriega L M. Alternativas terapéuticas en neumonía atípica. Rev Chil Infect 1996; 13: 104-9.

21.- Milla E, Zoógrafos L, Piguet B. Bilateral optic papillitis following Mycoplasma pneumoniae pneumonia. Ophthalmol 1998; 212: 344-6.

22.- Andrade C, Muñoz D, Koukoulis A, Martínez-Cueto P, Gómez Alonso J. Encephalopathy with focal lesions due to Mycoplasma pneumoniae. Neurology 1999; 14: $131-4$

23.- Cano A, Cano H, Fernández J, Artero J M, González L. Aseptic meningitis caused by Mycoplasma pneumoniae in a 19 year old woman. Eur J Clin Microbiol Infect Dis 1999; 18: 228-9.

24.- Cambonie G, Sarran N, Leboucq N et al. Mycoplasma pneumoniae meningoencephalitis. Arch Pediatr 1999; 6: 275-8.

25.- Smith R, Eviatar L. Neurologic manifestations of Mycoplasma pneumoniae infections: diverse spectrum of diseases. A report of six cases and review of the literature. Clin Pediatr (Phila) 2000; 39: 195-201.

26.- Fu M, Wong K S, Lam W W, Wong G W. Middle cerebral artery occlusion after recent Mycoplasma pneumoniae infection. J Neurol Sci 1998; 157: 113-5.

27.- Farraj R S, Mccully R B, Oh J K, Smith T F. Mycoplasma associated pericarditis. Mayo Clin Proc 1997; 72: 33-6.

28.- Koletsky R, Weinstein A. Case Reports. Fulminant Mycoplasma pneumoniae infection. Am Rev Resp Dis 1980; 122: 491-6.

29.- Fang G d, Fine M, Orlofff J et al. New and emerging etiologies for community-acquired pneumonia with implications for therapy. A prospective multicenter study of 359 cases Medicine 1990; 69: 307-16.

30.- Scully R E, Mark E J, Mcneely W F, Mcneely B V. Case records of the Massachusetts General Hospital. Case 5- 1992. N Engl J Med 1992; 326: 324-36.

31.- Berger R, Wadowsky R M. Rhabdomyolysis associated with infection by Mycoplasma pneumoniae: a case report. Pediatrics 2000; 105: 433-6.

Correspondencia a:

Maritza Navarrete C.

E-mail: maritzanavarrete@uach.cl 